\title{
6. Las metáforas espacio-temporales y la percepción del tiempo: un estudio comparativo sobre el español y el sueco
}

Emanuel Bylund \& Linn Andersson Konke

Stockholms universitet

\section{Introducción}

Desde la Antigüedad, la naturaleza del lenguaje y su relación con el pensamiento han sido objeto de especulaciones filosóficas y pesquisas científicas en un abanico de disciplinas, tales como la lingüística, la filosofía, la antropología y la psicología. Una pregunta central y controvertida en esta tradición concierne al papel que desempeña el lenguaje en la percepción del mundo y el modo de pensar. Formulada de forma más específica: la lengua que habla un individuo, ¿condiciona y da forma a su manera de pensar? En caso de ser afirmativa la respuesta, cabría entonces formularse una segunda pregunta: ¿acaso los hablantes de lenguas diferentes piensan asimismo de manera diferente?

En lo que sigue, se ilustrará esta última pregunta tomando como ejemplo el fenómeno del tiempo. El tiempo se define comúnmente como una magnitud física según la que se pueden ordenar sucesos pasados, presentes o futuros, y medir su duración o separación. El tiempo desempeña un papel fundamental en todos los niveles de nuestra vida cotidiana, como lo demuestran, por ejemplo, los instrumentos conceptualizados a lo largo de la historia para medirlo (v.gr. el reloj de sol y el reloj atómico). Un denominador común de los instrumentos de medición temporal es que su función gira en torno al movimiento, es decir, se basan en la dimensión espacial para conferir una medida de la dimensión temporal. De la misma forma, las lenguas del mundo muchas veces recurren de forma metafórica al dominio del

Cómo citar este capítulo:

Bylund, Emanuel \& Andersson Konke, Linn, Las metáforas espacio-temporales y la percepción del tiempo: un estudio comparativo sobre el español y el sueco. In: Engwall, Gunnel \& Fant, Lars (eds.) Festival Romanistica. Contribuciones lingüísticas Contributions linguistiques - Contributi linguistici - Contribuições linguísticas. Stockholm Studies in Romance Languages. Stockholm: Stockholm University Press. 20I5, pp. I I3-I30. DOI: http://dx.doi.org/IO.I6993/bac.f. License: CC-BY 
espacio para expresar relaciones temporales. Por ejemplo, el futuro por delante o tiempo atrás, o las palabras el porvenir y el pasado, las cuales hacen referencia a un movimiento metafórico del tiempo. Las lenguas difieren, con todo, en cuanto al tipo de metáfora espaciotemporal que usan (metáforas usadas para expresar relaciones temporales pero cuya semántica hace referencia al dominio espacial). Como se acaba de ver en los ejemplos anteriores, en español, así como en otras lenguas románicas y germánicas, se habla del pasado y del futuro en términos de 'delante' y 'detrás'. En otros idiomas, tal como el aymara, se habla del futuro en términos de detrás, y, del pasado en términos de delante, siguiendo la lógica de que el pasado, a diferencia del futuro, se conoce y es por lo tanto visible (es decir, podemos ver lo que está físicamente delante de nosotros pero no lo que está detrás). Sin embargo, no todas las lenguas utilizan un eje temporal horizontal para ordenar y situar los sucesos. En chino mandarín, por ejemplo, es común hacer referencia a un eje temporal vertical para este fin, según el que el pasado está hacia arriba y el futuro hacia abajo. En cada una de estas lenguas, las metáforas espacio-temporales obligan a sus hablantes a verbalizar las relaciones temporales de distintas maneras. $¿$ Es posible que al tenerse que expresar habitualmente de una forma determinada acerca del tiempo, el hablante acabe pensando sobre el tiempo de esa forma? ¿Acaso, por ejemplo, un hablante de aymara está más inclinado a comprehender el fenómeno del tiempo en términos horizontales que un hablante de chino? El tema de si la lengua tiene algún impacto en la forma en que aprehendemos el mundo, es decir, el tema de si los hablantes de lenguas diferentes piensan de manera diferente dadas las características propias de sus lenguas, se conoce con el nombre de relatividad lingüística.

El presente estudio se inscribe dentro del marco general de la relatividad lingüística. El objetivo general del estudio consiste en investigar, desde una perspectiva translingüística, la percepción del tiempo en hablantes de sueco y hablantes de español. Tomando como punto de partida las diferencias translingüísticas en lo que respecta a las metáforas espacio-temporales en español y en sueco, el estudio examina en particular los posibles efectos de dichas metáforas en la percepción de la duración temporal. Para conseguir este objetivo, el estudio se divide en dos subestudios: el primero tiene como fin estudiar las frecuencias distributivas de los diferentes tipos de metáforas espaciotemporales en español y en sueco, mientras que el segundo investiga en qué medida estos diferentes tipos de metáforas dan lugar a diferencias 
translingüísticas cognitivas, es decir, en qué medida producen diferencias en la estimación del tiempo por parte de suecohablantes e hispanohablantes.

El capítulo se organiza de la siguiente forma: en el apartado que viene a continuación, se da una breve vista panorámica de la investigación de la relatividad lingüística, y se sitúa el presente estudio dentro del marco teórico de las metáforas conceptuales. El apartado 'Estudio I' describe los objetivos y las delimitaciones del primer subestudio, cuyo fin es establecer el uso cuantitativo de las metáforas espacio-temporales. Este apartado comprende también un análisis estadístico, así como una interpretación de los patrones de uso de dichas metáforas. El siguiente apartado, 'Estudio II', presenta los objetivos, delimitaciones, y diseño experimental del segundo subestudio. En este apartado, se analizan las estimaciones de duración temporal por parte de hablantes de español y de sueco en una tarea psico-física. Se cierra el capítulo con unas conclusiones generales acerca de la relación entre las metáforas espaciotemporales y la percepción del tiempo.

\section{Trasfondo teórico}

\subsection{Relatividad lingüística}

En principio, la noción de relatividad lingüística se basa en dos premisas y una conclusión. La primera premisa presupone que las distintas lenguas en el mundo difieren fundamentalmente en cuanto a sus estructuras semánticas, y la segunda que la categorización semántica implicada en un idioma en concreto determina, bien de forma parcial o completa, el modo en que una persona categoriza, recuerda y percibe la realidad (Levinson \& Gumperz, I996). La conclusión lógica sacada a partir de estas dos premisas es que el modo en que una persona concibe la realidad varía en función de la lengua que habla. En vista de este razonamiento, puede formularse el silogismo de la relatividad lingüística:

I) Las lenguas que se hablan en el mundo caracterizan semánticamente la realidad de distintas maneras;

2) la estructura de una lengua determinada influye en la forma en que sus hablantes conciben la realidad;

3) por lo tanto, hablantes de diferentes lenguas conciben la realidad de manera diferente.

(Véase Whorf, I94I/I956) 
La antítesis del principio de la relatividad lingüística, es decir, que hablantes de diferentes lenguas comparten la misma concepción de la realidad, se fundamenta en los siguientes puntos: en primer lugar, presupone que la diversidad lingüística (i.e., las diferentes estructuras semánticas de las lenguas en el mundo) es una manifestación superficial, y que todas las lenguas comparten ciertos principios semánticos básicos (primitivos semánticos, Jackendoff, I990); en segundo lugar, conjetura que la estructura lingüística no hace más que proporcionar las formas fonológicas de conceptos preexistentes (bien innatos o adquiridos antes del lenguaje) (Fodor, I975). Partiendo de estos presupuestos, se deriva, pues, la conclusión de que la concepción de la realidad no varía en función de la lengua.

Durante los últimos lustros, el estudio sobre la relatividad lingüística ha experimentado un verdadero apogeo, sobre todo gracias al desarrollo teórico y metodológico de este campo de investigación, lo cual ha posibilitado refinadas operacionalizaciones de las nociones de 'pensamiento' y 'lenguaje'. En efecto, hoy en día, hay una variedad de estudios dedicados a indagar las posibles diferencias translingüísticas cognitivas en dominios perceptuales tales como el espacio, el tiempo, los colores, el movimiento y las sustancias (v.gr. Gentner \& Goldin-Meadow, 2003; Majid et al., 2004; Casasanto, 2008; Regier \& Kay, 2009).

\subsection{Las metáforas conceptuales}

Si bien es una concepción común que las metáforas pertenecen a un lenguaje poético o narrativo, estas aparecen también en el lenguaje cotidiano sin que nos demos cuenta de ello. En la prominente monografía de Lakoff \& Johnson (I980), se refuta la creencia tradicional de que el uso de la metáfora es una anomalía lingüística y que pertenece a un vocabulario especial. Al contrario, Lakoff \& Johnson ponen en evidencia que las metáforas son visibles en todo tipo de discurso, al decir, por ejemplo, esa camisa te va a costar un ojo de la cara es poco probable que el precio de una mercancía realmente fuera una parte del cuerpo, o al decir pásame el agua no se trata del líquido sino de la jarra o de la botella que contiene el agua, y está claro que no será posible interpretar el enunciado de forma literal. Lakoff y Johnson postulan que la metáfora, además de estar siempre presente en el lenguaje, también influye en nuestras acciones y pensamientos. De esta manera, el uso de las metáforas conceptuales es entendido como un proceso cognitivo, es decir, las metáforas residen en nuestro sistema conceptual, lo cual nos 
ayuda a entender conceptos abstractos y situaciones complejas. Con el fin de ilustrar qué es lo que constituye una metáfora conceptual presentamos a continuación algunos ejemplos (véanse Cuenca \& Hilferty, I999: I00; Lakoff \& Johnson, I980).

MORIR ES PARTIR

LAS PERSONAS SON ANIMALES LAS TEORÍAS SON EDIFICIOS
Nuestro padre nos ha dejado Él es muy burro Esa teoría carece de fundamentos empíricos LA ARGUMENTACIÓN ES UNA GUERRA
Juana atacó $\mathrm{mi}$ trabajo sobre la cognición

Si se analizaran las frases (en minúscula) una por una y sin contexto, serían todas expresiones metafóricas, es decir, casos individuales de una metáfora conceptual (en mayúscula). Por ejemplo, en la frase LA ARGU-MENTACIÓN ES UNA GUERRA, se emplean conceptos procedentes del dominio de la GUERRA para conceptualizar y hablar sobre el dominio de la ARGUMENTACIÓN, con lo cual la ARGUMENTACIÓN es estructurada y comprendida de la misma manera que la GUERRA.

Según Lakoff y Johnson, la metáfora conceptual está dividida en dominio origen y dominio destino. En el ejemplo de la metáfora conceptual LAS TEORÍAS SON EDIFICIOS, el sintagma LOS EDIFICIOS es el dominio origen y LAS TEORÍAS es el dominio destino. En otras palabras, al hablar de TEORÍAS se usa el mismo vocabulario que al hablar de EDIFICIOS. Lakoff \& Johnson ( I980) sostienen que la estructura de la metáfora conceptual sirve para comprender los conceptos abstractos: "the essence of a metaphor is understanding and experiencing one kind of thing in terms of another" (p. 5).

Para el establecimiento de la estructura de la metáfora conceptual se plantea el concepto de proyección (en inglés mapping). La proyección indica la relación entre el dominio origen y el dominio destino y se divide en dos tipos: correspondencias ontológicas y correspondencias epistémicas. La correspondencia ontológica tiene como función poner de relieve las relaciones analógicas existentes entre las partes más importantes de cada dominio, en otras palabras, conecta subestructuras entre los dos dominios. La correspondencia epistémica, a su vez, describe los factores comunes entre los dominios y traslada información desde el dominio origen al dominio destino. En los siguientes ejemplos 
se emplea la metáfora conceptual LAS IDEAS SON ALIMENTOS (Cuenca \& Hilferty, I999: I02):

\section{Correspondencia ontológica:}

a. las ideas corresponden a los alimentos

b. la persona que come los alimentos corresponde a la persona que acepta la idea

c. cocinar los alimentos corresponde a concebir las ideas

\section{Correspondencia epistémica:}

Dominio origen: los alimentos sustentan el cuerpo

Dominio destino: las ideas sustentan la mente

\subsection{Diferencias translingüísticas en la percepción del tiempo}

Una idea básica de Lakoff \& Johnson (I980) es, pues, que las proyecciones de las metáforas conceptuales sirven para entender conceptos abstractos. Un ejemplo de tal concepto es el tiempo, el cual, como se pudo ver más arriba, se subdivide en varias metáforas conceptuales. La metáfora conceptual EL TIEMPO ES UN OBJETO MOVIBLE es la que se utiliza al expresar tanto sucesión (v.gr. el lunes viene antes del martes) ${ }^{\mathrm{I}}$ como duración (v.gr. una reunión corta), conceptualizando de esta manera el tiempo como un objeto que se mueve a través del espacio.

Las lenguas del mundo exhiben cierta variación en las metáforas conceptuales que usan para expresar duración temporal. En español, por ejemplo, normalmente se habla de duración en términos de cantidad, a saber, mucho tiempo y poco tiempo. La duración se expresa, pues, de la misma manera que el espacio, por ejemplo, mucho vino y poca agua. El griego exhibe un patrón muy similar: en esta lengua, se recurre a metáforas de cantidad para hacer referencia a duración, por ejemplo, poli ora ('mucho tiempo'), e incluso en contextos en que el español hace uso de metáforas de distancia para denotar duración, como por ejemplo, una reunión larga, el griego usa metáforas de cantidad: megali synandisi (literalmente 'reunión grande', es decir, 'reunión larga'). Las lenguas germánicas, por otro lado, normalmente utilizan metáforas basadas en distancia para expresar duración. En inglés, por ejemplo, la expresión long time ('tiempo largo') es comúnmente usada, al igual que en afrikaans, lank tyd ('tiempo largo').

Tomando como punto de partida estas diferencias translingüísticas, Casasanto et al. (2005) investigaron, en primer lugar, la frecuencia de diferentes tipos de metáforas espacio-temporales en español, griego, inglés e indonesio, y en segundo lugar, la percepción del tiempo en 
hablantes de estas lenguas. Un análisis de la frecuencia distributiva de las metáforas mostró que en inglés e indonesio había una marcada preferencia a usar long time ('largo tiempo') y waktu panjang ('largo tiempo'), respectivamente, a diferencia de much time ('mucho tiempo') y waktu banyak ('mucho tiempo'). En español y griego, por el contrario, la tendencia iba a la inversa, en el sentido de que las expresiones mucho tiempo y poli ora ('mucho tiempo') exhibían una frecuencia considerablemente más alta que largo tiempo y makry kroniko diatstima ('largo tiempo'). En otras palabras, en español y en griego, predominaban las metáforas espacio-temporales de cantidad, mientras que en inglés y en indonesio, las metáforas de distancia eran más frecuentes.

Habiendo establecido las diferencias translingüísticas, Casasanto et wal. (2005) estudiaron la percepción de la duración por parte de hablantes de estas lenguas mediante una tarea de estimación temporal. En esta tarea, los participantes veían o bien líneas o contenedores que iban creciendo o llenándose en la pantalla, y luego tenían que estimar el tiempo que estas animaciones tardaban en crecer. Los resultados de este experimento mostraron que los hablantes de griego y de español se dejaron influir por el crecimiento de los contenedores, en el sentido de que cuanto más se llenaban estos, más larga les parecía la duración a los hablantes de estas lenguas. En cambio, no se registró el mismo efecto en el caso de las estimaciones de duración de las líneas. Los análisis de las estimaciones hechas por los hablantes de inglés y de indonesio evidenciaron el patrón opuesto: mientras que estos hablantes no se vieron influidos por el nivel de los contenedores, sí que se dejaron llevar por la longitud de las líneas, creyendo que cuanto más crecían estas, más tiempo les había transcurrido. En vista de estos resultados, Casasanto et al. concluyeron que en el dominio perceptual del tiempo, las metáforas espacio-temporales idiosincrásicas de un idioma pueden dar lugar a que los hablantes conciban la duración temporal de una forma correspondiente a esas metáforas. Esto, a su vez, implica que hablantes de lenguas con diferentes tipos de metáforas espacio-temporales conciben el tiempo de formas distintas (véase también Casasanto, 2008).

\section{Estudio I}

\subsection{Objetivos y delimitaciones}

¿Cuáles son los tipos de metáforas espacio-temporales y su uso en sueco y en español? La investigación de Casasanto nos proporciona una respuesta parcial a esta pregunta, por lo menos en lo que respecta a la lengua española (véase más arriba). Los patrones de uso 
de las metáforas espacio-temporales en sueco y su grado de similitud con los patrones de uso en español son temas que, sin embargo, se están aún por investigar. El objetivo del Estudio I consiste, pues, en establecer y comparar las frecuencias distributivas de los principales tipos de metáforas espacio-temporales de distancia y cantidad en sueco y en español. Este procedimiento constituye, sin duda, un paso central en la búsqueda de diferencias cognitivas translingüísticas en la percepción del tiempo: en caso de que no se registren diferencias translingüísticas en los patrones de uso de las metáforas espaciotemporales en español y sueco, hay poca base para creer que los hablantes de español y de sueco conceptualizan el tiempo de manera diferente. Por otro lado, si resulta que estas lenguas difieren a este respecto, hay motivo para indagar si tales diferencias originan diferentes percepciones del tiempo.

Como queda dicho, el Estudio I tiene por objeto examinar y comparar las frecuencias distributivas de las metáforas espacio-temporales de español y de sueco. El estudio tiene, en otras palabras, un enfoque principalmente de índole cuantitativa. Siguiendo la investigación de Casasanto et al. (2004), el presente estudio examina las frecuencias distributivas de expresiones tales como largo tiempo y mucho tiempo. Mientras que el estudio de Casasanto et al. midió las frecuencias de expresiones que denotan una duración temporal mayor (v.gr. mucho tiempo en español, y long time en inglés), este estudio aspira a investigar también expresiones que hacen referencia a una duración de menor magnitud (v.gr., poco tiempo y corto tiempo).

\subsection{Materiales y método}

Conforme a la metodología de Casasanto et al. (2004), el presente estudio hizo uso del buscador Google para calcular las frecuencias distributivas de las metáforas espacio-temporales en las lenguas bajo investigación. Este procedimiento consiste simplemente en calcular el número de entradas de las expresiones relevantes de duración temporal de distancia y cantidad (véase Tabla I).

Tabla 1. Las expresiones analizadas en el estudio I

\begin{tabular}{lll}
\hline & Distancia & Cantidad \\
\hline Español & largo tiempo, corto tiempo & mucho tiempo, poco tiempo \\
Sueco & lang tid, kort tid & mycket tid, lite tid \\
\hline
\end{tabular}


Asimismo, los números de entradas de estas expresiones fueron agrupados en una tabla de contingencia en diferentes combinaciones y analizados mediante un test de chi cuadrado $\left(\chi^{2}\right)$.

\subsection{Resultados y discusión}

Los análisis se dividen en tres etapas. La primera atañe a la distribución de expresiones de duración de mayor magnitud (lo cual es el mismo tipo de análisis que realizaron Casasanto et al.). Los resultados de este análisis se presentan en la tabla 2.

Tabla 2. Frecuencias distributivas de metáforas de mayor duración temporal

\begin{tabular}{lrr}
\hline & Distancia $($ largo, lång) & Cantidad (mucho, mycket) \\
\hline Español & 5860000 & 60900000 \\
Sueco & I8 800000 & 3070000 \\
\hline
\end{tabular}

Un análisis de chi cuadrado demuestra que la distribución de los diferentes tipos de metáforas varía según la lengua de forma significativa, $\chi^{2}=4,08 \times \mathrm{xI}^{5}$, grados de libertad $(g l)=\mathrm{I}, p<0,000 \mathrm{I}$. Esta variación inter-grupal exhibe una magnitud intermedia, $d=0,49$.

El segundo análisis concierne a la distribución de expresiones de duración temporal de magnitud menor, y difiere, por lo tanto, del enfoque de Casasanto, el cual concernía solamente a las expresiones de mayor duración. Los resultados de las frecuencias distributivas de estas expresiones se presentan en la Tabla 3.

Tabla 3. Frecuencias distributivas de metáforas de menor duración temporal

\begin{tabular}{lrr}
\hline & Distancia $($ corto, kort $)$ & Cantidad (poco, lite) \\
\hline Español & 2700000 & 32200000 \\
Sueco & 8950000 & 2990000 \\
\hline
\end{tabular}

$\mathrm{Al}$ igual que en el análisis anterior, la variación translingüística de las expresiones espacio-temporales de magnitud menor es estadísticamente significativa, $\chi^{2}=2$, I $5 \mathrm{XIO}^{5}, g l=\mathrm{I}, p<0,000 \mathrm{I}$. En el caso de estas expresiones, la magnitud de la variación resulta incluso más robusta, $d=\mathrm{I}, 84$. 
La tercera etapa del presente análisis implica sumar las frecuencias de las tablas 2 y 3 para formar macrocategorías de metáforas de distancia y cantidad de español y de sueco, sin tener en cuenta su referencia a la magnitud de duración temporal. Como es de esperar, la distribución que emerge a raíz de este procedimiento exhibe una significación y magnitud muy sólidas, $\chi^{2}=7,02 \times 10^{6}, g l=\mathrm{I}, p<0,000 \mathrm{I}, d=2,07$. Estos resultados de frecuencia, convertidos en porcentajes, se presentan en el Gráfico I.

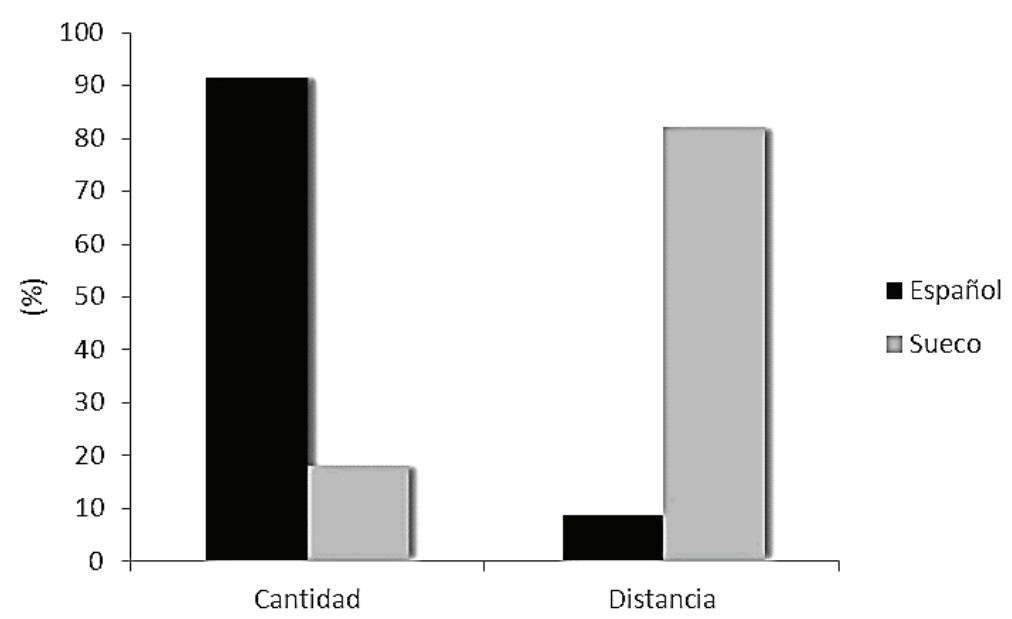

Gráfico 1. La distribución de expresiones de duración de cantidad y distancia en español y en sueco

A modo de resumen, se puede constatar que hay una diferencia translingüística muy saliente en el uso de las metáforas espacio-temporales en las lenguas bajo investigación: en sueco prevalecen las expresiones basadas en distancia (lång tid y kort tid), mientras que en español, las expresiones de cantidad (mucho tiempo y poco tiempo) son las más frecuentes. Cabe destacar, sin embargo, que por muy salientes que sean estas preferencias, existe en ambas lenguas la posibilidad de hablar del tiempo usando ambos tipos de metáforas. En sueco, para empezar, un I $8 \%$ de las expresiones analizadas conceptualizan el tiempo en términos de cantidad, v.gr., 'mycket tid'. Es importante hacer constar, no obstante, que la expresión mycket tid denota otro tipo de valor temporal: a diferencia de lang tid, mycket tid no hace referencia a duración temporal, sino que muchas veces denota tiempo invertido, repetido, o acumulado, v.gr., jag har ägnat mycket tid àt X ('he dedicado mucho tiempo a X'). 
Asimismo, en español, existe la posibilidad de usar metáforas de distancia (v.gr., largo tiempo) a la hora de hablar del tiempo. Tal como muestran los resultados, este tipo de metáforas representa un $8 \%$ del material analizado. ¿Cuál es, entonces, la diferencia entre las expresiones mucho tiempo y largo tiempo? De forma preliminar, puede decirse que la expresión de largo tiempo se usa con el fin de describir un período determinado de duración extraordinariamente larga. De hecho, la expresión largo tiempo aparece a menudo en la frase un largo tiempo.

El objetivo de Estudio I ha sido establecer las frecuencias distributivas de las metáforas espacio-temporales en español y en sueco, tal y como han sido definidas y operacionalizadas en Tabla I. Los análisis estadísticos han puesto en evidencia que la distribución de metáforas de distancia y de cantidad varía en función de la lengua. Los resultados confirman, pues, en parte los hallazgos de Casasanto et al. (2004), quienes encontraron un patrón de uso muy similar para el español. Cabe reconocer, con todo, que el método usado en este estudio y en el de Casasanto impone ciertas limitaciones, en lo que respecta tanto a la fiabilidad como a la validez de los resultados. En primer lugar, el material analizado no proviene de un corpus lingüístico muy controlado, sino que se basa simplemente en el número de entradas que las expresiones investigadas produjeron en Google. A pesar de que el elevado número de entradas ofrece una potencia estadística considerablemente alta, están muy reducidas las herramientas para controlar parámetros como, por ejemplo, género, puntuación y orden de palabras. ${ }^{2}$ En segundo lugar, el enfoque adoptado, limitado a las expresiones de mucho tiempo, lång tid, etc., implica hacer caso omiso de otros recursos como, por ejemplo, los adverbios temporales, que ofrecen las lenguas bajo investigación para hablar del tiempo. Volveremos a tocar este tema en las conclusiones generales.

\section{Estudio II}

\subsection{Objetivos y delimitaciones}

Habiendo establecido los principales patrones de uso de metáforas espacio-temporales en español y en sueco, cabe preguntarse: ¿cuáles son las consecuencias cognitivas de esta variación translingüística? Es decir, el hecho de que un hispanohablante normalmente hable de duración en términos de cantidad, ¿significa que esta persona piensa 
o comprende el fenómeno del tiempo en base a este tipo de términos espaciales? Igualmente, ¿concibe el suecohablante el tiempo en términos espaciales de distancia por el hecho de usar tales términos al hablar sobre el mismo? Siguiendo los postulados de la teoría de las metáforas conceptuales, la respuesta a estas preguntas sería afirmativa. El Estudio II va más allá e intenta investigar este tema de forma empírica, planteando la siguiente pregunta de investigación: ¿surgen diferencias translingüísticas en la percepción de la duración temporal por parte de suecohablantes e hispanohablantes, a raíz de los diferentes patrones de uso de metáforas espaciales que exhiben estas lenguas?

Para proporcionar una respuesta a esta pregunta, el presente estudio investigará en qué medida la percepción del tiempo se ve influida por diferentes tipos de información espacial. Siguiendo la técnica utilizada por Casasanto et al. (2004), el presente estudio se limita a medir la interferencia espacial a partir de estímulos visuales que representan distancia y cantidad. Partiendo de los resultados presentados por Casasanto et al. (2004), puede formularse la siguiente hipótesis: la percepción de la duración por parte de los suecohablantes se verá interferida por los estímulos de distancia, mientras que en el caso de los hispanohablantes, la interferencia se producirá por parte de los estímulos de cantidad. En otras palabras, los suecohablantes pensarán que cuanto más crece una línea, más tiempo ha pasado, mientras que los hispanohablantes pensarán que cuanto más se llena un contenedor, más tiempo ha transcurrido.

A diferencia del estudio de Casasanto, que concernía también la estimación de información espacial, el presente estudio está enfocado solamente en la estimación de la duración.

\subsection{Participantes}

Diez hablantes nativos de sueco y ro hablantes nativos de español participaron en el estudio. Los hablantes de español o bien estaban de visita en Suecia en la fecha en que se realizaba el experimento, o bien se habían mudado recientemente a Suecia -de todas formas sus conocimientos de la lengua sueca eran inexistentes o muy rudimentarios. Los suecohablantes no tenían conocimientos de la lengua española. Los participantes tenían entre 2 I y 45 años de edad, y cada uno de ellos había estudiado -o estaba estudiando- en la universidad. Recibieron 50 coronas suecas (aprox. $5 €$ ) como remuneración por su participación. 


\subsection{Materiales y método}

Un total de 98 animaciones fueron creadas en PowerPoint. Estas animaciones consistían en 49 líneas que iban creciendo horizontalmente de izquierda a derecha, y 49 rectángulos (o 'contenedores') que se iban llenando de negro verticalmente desde abajo hacia arriba. El crecimiento de las animaciones variaba entre 5 y I $7 \mathrm{~cm}$, con un aumento de $2 \mathrm{~cm}$ (es decir, $5 \mathrm{~cm} ; 7 \mathrm{~cm} ; 9 \mathrm{~cm}$; I I cm, etc.). La duración de las animaciones oscilaba entre I y 5 segundos, con un aumento de aproximadamente 0,67 segundos (es decir, I,०0 s; I,67 s; 2,33 s; 3,00 s, etc.). Había, pues, 7 duraciones diferentes y 7 crecimientos diferentes. Cada una de las duraciones aparecía con cada uno de los crecimientos, creando así un total de 49 líneas y 49 contenedores. Este diseño difiere del de Casasanto et al., en el sentido de que aquí se mezclaron los estímulos de líneas y contenedores, mientras que en el estudio de Casasanto et al. se presentaron estos estímulos por separado (v.gr., un grupo de ingleses hizo la tarea de líneas, mientras que otro grupo de ingleses hizo la de contenedores).

Se presentaron las animaciones en series aleatorias en una pantalla de I 7 pulgadas de I $280 x$ I0 24 píxeles. La tarea de los participantes consistía en estimar la duración de cada animación, haciendo uso del ratón para simular el tiempo que tardaba cada animación en crecer. En concreto, esta tarea implicaba hacer clic para marcar el comienzo de la duración percibida, esperar el tiempo estimado, y hacer otro clic para marcar el final. Se registraron las respuestas de los participantes mediante el programa Time Station. Antes de que se pusiera en marcha el experimento, los participantes recibieron instrucciones detalladas acerca de la tarea. Estas instrucciones carecían de pistas lingüísticas que pudieran influir o sesgar las estimaciones de los participantes (v.gr. en sueco: uppskatta hur lång tid..., literalmente 'estima cuán largo tiempo...’').

\subsection{Resultados y discusión}

El análisis se realizó en dos pasos: en primer lugar, se examinó el grado de acierto de las estimaciones de duración de los dos grupos; y en segundo lugar, se analizó en qué medida los participantes se vieron influidos por los crecimientos de las animaciones a la hora de estimar el tiempo, es decir, el grado de interferencia espacial. El primer paso analítico es primordial: si no hay diferencias inter-grupales en la estimación de la duración, las diferencias que se observen a nivel de interferencia espacial no pueden deberse a la existencia de diferencias generales en la capacidad de hacer estimaciones de duración. 
Con el fin de analizar las estimaciones de duración temporal de los grupos de participantes, se calcularon las medias de las estimaciones por cada duración, independientemente del crecimiento, en cada uno de los grupos. Una vez obtenidas las medias, se realizó un test de regresión entre las duraciones estimadas y las verdaderas. Los resultados de este test demostraron que tanto el grupo de hispanohablantes como el grupo de suecohablantes mostraron una estimación del tiempo muy acertada, $r^{2}=0,99, g l=7$, inclinación $($ incl $)=0,8 \mathrm{I}, \mathrm{y} r^{2}=0,99, g l=7$, $i n c l=0,83$, respectivamente. Los dos grupos no se diferenciaron, pues, en su estimación general del tiempo.

El segundo paso del análisis consistió en medir la posible interferencia espacial en las estimaciones de duración hechas por los dos grupos. Empezando por los hispanohablantes, una regresión lineal de las duraciones estimadas de cada crecimiento mostró que las estimaciones de duración de los contenedores se veían influidas por el desplazamiento de los mismo, $r^{2}=0,98, g l=6$, incl $=4,24$. Este efecto exhibió una significación estadística relativamente alta, $p<$ o, О I. Resultó, no obstante, que también había interferencia espacial en las estimaciones de las duraciones de las líneas, $r^{2}=0,94, g l=6$, incl $=3,99, p>0$, О I. En otras palabras, se registraron efectos de interferencia espacial tanto en el caso de los contenedores como en el de las líneas. Los análisis de interferencia espacial de los suecohablantes pusieron en evidencia un patrón muy similar: se observó un efecto general de crecimiento en las estimaciones de duración tanto de las líneas como de los contenedores, $r^{2}=0,8 \mathrm{I}, g l=6, i n c l=2,22, p<0,0 \mathrm{I}, \mathrm{y} r^{2}=0,94, g l=6$, incl = 2,70, $p<0, \mathrm{O}$, respectivamente. Estas regresiones se representan en los diagramas de dispersión en los Gráficos 2.I-2.4. A modo de resumen, los análisis llevados a cabo hasta ahora han evidenciado que había efectos de interferencia espacial, independientemente del tipo de estímulo, en ambos grupos.

Con el fin de comprobar la constancia de estos resultados, se siguió el procedimiento del estudio de Casasanto (2005), en el que se excluyeron del análisis los estímulos más extremos (a saber, la máxima y mínima duración, y el máximo y mínimo crecimiento). Mientras que Casasanto eliminó cuatro medidas de esta forma, el presente estudio se limita a eliminar solamente dos, puesto que una eliminación de cuatro hubiera reducido el número total de medidas a tres, lo cual se considera muy poco para una regresión. Los resultados de este análisis mostraron, sin embargo, un patrón muy parecido al resultado más arriba: hispanohablantes, contenedores: $r^{2}=0,93, g l=4$, incl $=3,59, p<0,0 \mathrm{I}$; 
líneas: $r^{2}=0,88, g l=4$, incl $=4,37, p<0,02$; suecohablantes, contenedores: $r^{2}=0,88, g l=4, i n c l=2,75, p<0,02$; líneas: $r^{2}=0,88, g l=4$, incl $=\mathrm{I}, 67, p<0,02$.

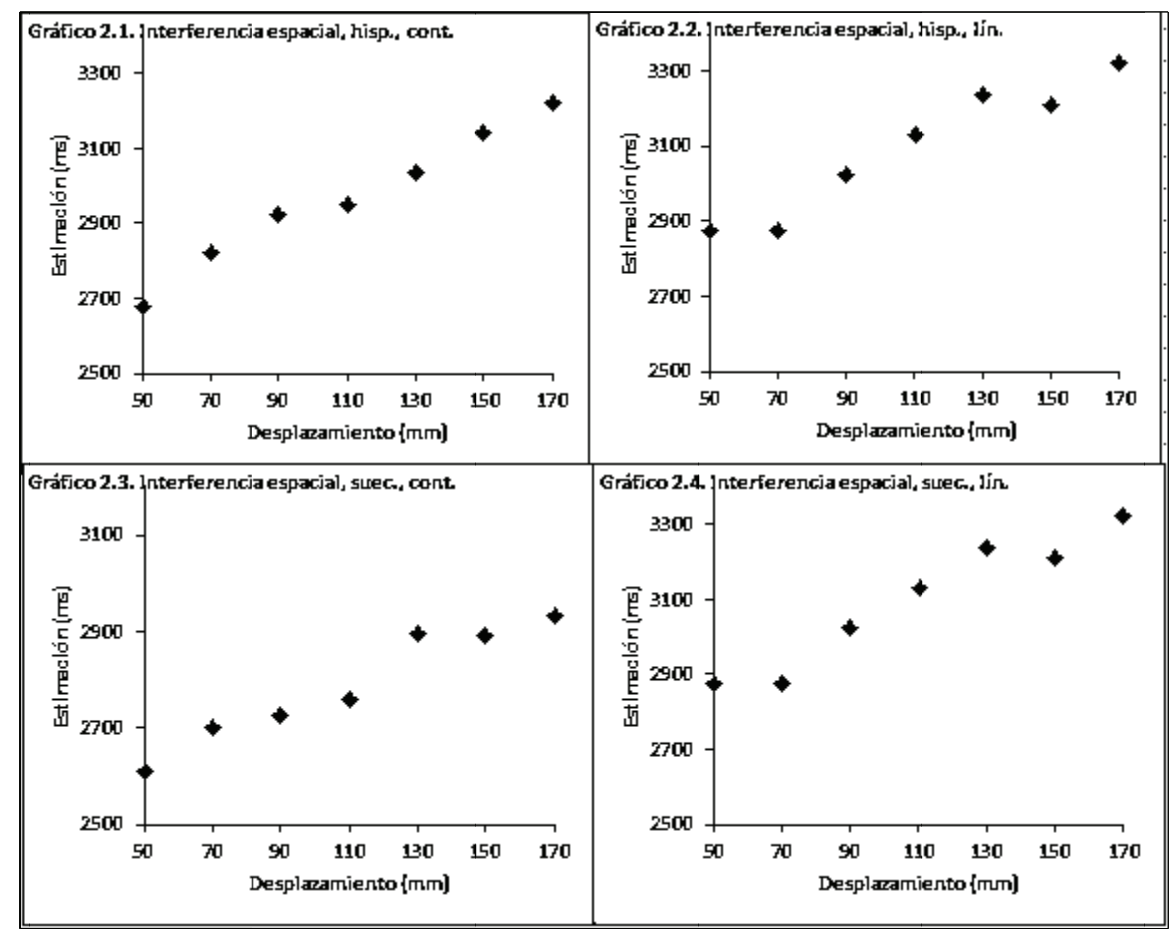

Gráficos 2.1-2.4. Diagramas de dispersión

Los resultados muestran, pues, que incluso con esta modificación del análisis los hablantes de español y de sueco exhiben el mismo tipo de interferencia espacial. Al interpretar estos resultados, cabe tener en cuenta que el presente diseño difiere del de Casasanto et al. (2004) en un aspecto importante: en aquel estudio, los participantes vieron los estímulos de contenedores y líneas por separado, mientras que en el presente estudio los participantes vieron estos estímulos mezclados. Los resultados de este estudio no rechazan, en otras palabras, los hallazgos de Casasanto et al. (2004), pero sí modifican las conclusiones que sacan estos investigadores acerca del lenguaje y su relación con la percepción temporal. Si bien las metáforas espacio-temporales influyen en la percepción de la duración, tal como muestra el estudio de Casasanto, la incidencia de esta influencia está limitada a ciertas condiciones: si el participante tiene que estimar la duración a partir de un solo tipo de estímulo (es decir, o contenedores, o líneas), se producen diferencias 
translingüísticas conformes a las metáforas espacio-temporales idiosincrásicas de la lengua del participante. No obstante, si el participante se enfrenta a la tarea de estimar la duración a partir de dos tipos de estímulos diferentes, las diferencias translingüísticas desaparecen. Posiblemente esto se debe a que la influencia de cierto tipo de estímulo (v.gr. líneas) en la percepción temporal es transferida a la percepción de otro tipo de estímulo (v.gr. contenedores). Es decir, al participante le resulta difícil dejarse influir por solo un tipo de información espacial, y al mismo tiempo hacer caso omiso a otro tipo de información espacial.

\section{Conclusiones}

El objetivo principal del presente estudio ha sido indagar, desde una perspectiva translingüística, la relación entre las metáforas espaciotemporales y la percepción del tiempo. Los resultados del primer subestudio pusieron en evidencia que en sueco hay una preferencia destacada por usar metáforas de distancia para referirse a la duración, mientras que en español prevalece el uso de las metáforas de cantidad. El segundo subestudio, que era de corte experimental, mostró que no había diferencias en la percepción del tiempo entre hablantes de español y hablantes de sueco, en el sentido de que ambos grupos se vieron influidos por la información espacial -independientemente del tipo de dicha información- en una tarea consistente en estimar la duración de diferentes animaciones. El hecho de que estos resultados no converjan con los de Casasanto et al. (2004) tiene, como se mencionaba más arriba, implicaciones para la interpretación de los efectos del lenguaje en el pensamiento. En general, estos resultados están en consonancia con estudios anteriores en los que se muestra que la influencia del lenguaje sobre el pensamiento no es un fenómeno de todo o nada, sino que está limitada a ciertos procesos cognitivos realizados bajo ciertas condiciones (para una discusión más detallada, véase Athanasopoulos \& Bylund, 2013).

Asimismo, los resultados del presente estudio también son relevantes para la teoría de las metáforas conceptuales. Dado que no se registraron efectos de interferencia espacial que fueran atribuibles a las metáforas espacio-temporales prevalentes en los idiomas estudiados, cabe preguntarse en qué medida se puede sustentar la idea de que las metáforas conceptuales constituyen una herramienta importante para aprehender un concepto abstracto como el tiempo, tal y como postulan Lakoff $\&$ Johnson (I980). Los hallazgos del presente estudio no descartan, desde 
luego, la noción básica de las metáforas conceptuales, pero sí ponen en duda el supuesto que guía este marco teórico. Según este supuesto, el nivel semántico equivale al nivel conceptual, es decir, se basa en la semántica de una expresión lingüística para explicar cómo un individuo entiende o aprehende un determinado concepto. Está claro que un análisis semántico de una estructura lingüística constituye un paso primordial en el camino hacia una teoría del pensamiento y del lenguaje, pero este paso de por sí no es suficiente para explicar o sacar conclusiones acerca de cómo un individuo piensa acerca de un concepto dado -para entender esto, haría falta evidencia empírica del comportamiento cognitivo.

\section{Notas}

I. También cabe notar que el adverbio antes, si bien tiene un significado principalmente temporal en el español moderno, originalmente deriva del latín ante, lo cual significaba 'delante' o 'anterior' (cf. la preposición español ante).

2. Desafortunadamente, todavía no existen corpora de sueco y español comparables o paralelos como para llevar a cabo un estudio de corpus de este tipo.

\section{Referencias}

Athanasopoulos, Panos \& Emanuel Bylund. 20I3. "Does grammatical aspect affect motion event cognition? A cross-linguistic comparison of English and Swedish speakers”. Cognitive Science, 37. 286-309.

Casasanto, Daniel. 2005. Perceptual foundations of abstract thought. Tesis doctoral. Cambridge, MA: MIT.

Casasanto, Daniel. 2008. "Who's afraid of the big bad Whorf? Crosslinguistic differences in temporal language and thought". Language Learning, 58, Supplement I. 63-79.

Casasanto, Daniel \& Lera Boroditsky. 2008. "Time in the mind: Using space to think about time”. Cognition, I06. 579-593.

Casasanto, Daniel \& Lera Boroditsky, Webb Phillips, Jesse Green, Shima Goswani, Simon Bocanegra-Thiel et al. 2004. "How deep are the effects of language on thought? Time estimation in speakers of English, Indonesian, Greek, and Spanish". Proceedings of the Annual Conference of the Cognitive Society, 26. I86-I9I.

Cuenca, María Josep \& Josep Hilferty. I999. Introducción a la lingüística cognitiva. Barcelona: Ariel. 
Fodor, Jerry. 1975. The language of thought. Nueva York: Crowell.

Gentner, Dedre \& Susan Goldin-Meadow (eds.). 2003. Language in mind: Advances in the study of language and thought. Cambridge, MA: MIT Press.

Gumperz, John J. \& Stephen Levinson. I996. “Introduction: linguistic relativity re-examined”. In: Gumperz, John \& Stephen Levinson (eds.), Rethinking linguistic relativity. Cambridge: Cambridge University Press. I-I 8.

Jackendoff, Ray. I990. Semantic structure. Cambridge, MA: MIT Press.

Lakoff, George \& Mark Johnson. I980. Metaphors we live by. Chicago: University of Chicago Press.

Majid, Asifa, Melissa Bowerman, Sotaro Kita, Daniel Haun \& Stephen Levinson. 2004. "Can language restructure cognition? The case for space”. Trends in Cognitive Sciences, 8.108-I I4.

Pinker, Steven. I994. The language instinct. London: Penguin books.

Regier, Terry \& Paul Kay. 2009. "Language, thought and color: Whorf was half right". Trends in Cognitive Sciences, I3. 439-446.

Whorf, Benjamin L. I94I/2000. Language, thought and reality: selected writings of Benjamin Lee Whorf. Cambridge: MIT Press. 